\title{
A Priority-Based MAC Protocol for the Terahertz In- Vivo Nano-Networks in the Presence of Noise
}

\author{
Jiali Kan, Juan Xu, Yakun Zhao, Jiaolong Jiang and Yan Zhang \\ College of Electronics and Information Engineering Tongji University Shanghai, China
}

\begin{abstract}
Advances in nanotechnology and sensor networks recent years provide the opportunity for developing Wireless NanoSensor Networks (WNSNs) in the terahertz (THz) band(0.110THz). It's a new challenge for the THz nano-networks to satisfy biomedical applications with a medium access control (MAC) protocol to support prioritized services for various traffics in human body. In addressing this challenge, we proposed a priority-based MAC (PB-MAC) protocol for electromagnetic (EM) communication over THz bands for in-vivo nano-networks, which takes the channel noise within two human tissues into account. The PB-MAC protocol ensures medical data with different kinds of priorities sorted into priority order by utilizing a new priority-queue(PQ) algorithm. Network Simulator 3 (NS3) implemented simulations, and the results demonstrate that the PB-MAC protocol guarantee priority classified data received by priority, and is efficient in terms of the average transmission delay and network throughput compared with the transparent transmission MAC protocol.
\end{abstract}

Keywords—priority; MAC; in-vivo nano-networks; noise model; terahertz channel

\section{INTRODUCTION}

In vivo wireless nanosensor network (iWNSN) [1] is composed of nano-scale communication devices, which can operate in real time inside the human body. In vivo health monitoring system and drug delivery applications are the basis of iWNSN. Recent studies have shown that the development of novel materials such as carbon nanotubes (CNT) and graphene enables nanoscale communications in the terahertz THz band inside the human body as nano-antennas [2-4]. Due to the fact that nano-devices are restricted with limited communication capabilities, it's the optimal choice to utilize Time Spread On-Off Keying (TS-OOK) as the modulation scheme to ensure nano-devices communicate at the $\mathrm{THz}$ band [5]. Based on the channel noise model proposed in [6], the feasibility of TS-OOK for the $\mathrm{THz}$ communication in human body is verified by measuring noise power and receiving power as a function of transmission distance [7].

Reference [8] holds the view that medical application signals which are life critical possess the rigorous delay requirements. Thus it is required that a MAC protocol with specific functions should satisfy the needs of medical applications. IEEE 802.15.4 MAC protocol can't guarantee differentiated data services on account of all signals are treated equally in the superframe.

Based on the requirements in health detection system, some priority-based schemes for data transmission are proposed. Reference [9] proposes a prioritized IEEE 802.15.4 for Wireless Sensor Network (WSN) with explicit priority mechanism to finish the delivery of prioritized packet without the assumption that all the nodes are within the transmission range of each other. A priority based MAC called Urgencybased MAC (U-MAC) is proposed in [10] for Wireless Body Area Networks (WBANs) which gives higher priority to sensor nodes sending urgent health data. The U-MAC have the capability of providing differentiated services and limitation of ignoring the different QoS needs among lower sensor nodes. And [11] proposes a priority-guaranteed MAC protocol which ensures consumer electronic and medical information are transferred on specified channel to achieve differentiated service which is divided into 2 kinds of priorities without considering the urgent signals.

In this paper, we proposed a priority-based MAC (PB-MAC) protocol which classifies data traffics including common data and urgent data of the terahertz in-vivo nano-networks into 3 priorities. The PB-MAC protocol can ensure the transferred data with higher priority be received first by using a new Priority-Queue algorithm (PQ) which sorts data by priority. The rest of this paper is organized as follows. In Section II, the system model is introduced, including the data priority classification and the nano-network model. The proposed PBMAC protocol is described in Section III. In Section IV, we define two evaluation indicators: average transfer delay and average throughput to evaluate the performance of the new PBMAC protocol. And then, we demonstrate and analyze simulation results for the performance of PB-MAC protocol in Section V. At last, we make a conclusion of this paper in Section VI.

\section{SYSTEM MODEL}

\section{A. Data Priority Classification}

Among the health monitoring systems of iWNSNs, we have to take emergency physiological parameters which is lifecritical like heart rate into consideration [12], thus, it should be considered to be the most important data and have the highest priority in health monitoring system. Then, medical information with normal vital signals like Pulse Oxygen Saturation (SpO2) and electrocardiogram (ECG) and so on, is less important and ranks the second while discontinuous medical information which require periodic measurement like temperature and blood pressure ranks the last. In short, we briefly classify data into 3 priorities in this paper shown in Table 1. 
TABLE I. DATA PRIORITY

\begin{tabular}{|c|c|c|}
\hline Priority level & Data type & Examples \\
\hline 1 & P1 & Life-critical signals \\
\hline 2 & P2 & ECG/ SpO2 \\
\hline 3 & P3 & Temperature/blood pressure \\
\hline
\end{tabular}

B. Nano-Network Model

We adopted one-hop star style as the nano-network topology, $\mathrm{N}$ nano-nodes fixed randomly within a circle of radius $\mathrm{r}$ inside two human tissues (fat and skin). One nanomicro interface (nano-interface) locates at the center of the circle, and aggregates information transmitted by nano-nodes. All nano devices are in each other's communication range. Every nano device has its own ID. Nano-interface's ID is N, nano-nodes' IDs increase continuously from 0 . Similarly, all packets generated by nano-nodes in this nano-network also have IDs increase from 0 chronologically. In the case of random distribution of nano-nodes, for simplicity, we defined each nano-node can transmit data with three priorities.

\section{PB-MAC PROTOCOL}

PB MAC protocol is proposed to satisfy the requirements of different priorities services in medical monitoring system. In medical application, the data are usually periodic, and the health indicators related to life safety should be presented first which means that this kind of data have the highest priority. In order to simulate real application scenario, this paper assumes that the packet intervals of nano-nodes are the same. And the priority-queue (PQ) algorithm is designed with the following principals:

- Higher priority packets are always sent first than those with lower priority under any circumstances.

- The average transmission delay of each packet in the channel is equal.

The PQ algorithm proposed in this paper is based on priority queue. In the priority queue, all elements are sorted by priority. Specifically, each time an element enters a queue, the queue is prioritized with the highest priority at the top and the lowest priority at the bottom. Meanwhile, we can only acquire elements from the top to the bottom according to priority. We adopt priority queue based on minimum heap which sorts elements into ascending order in our PQ algorithm. The minimum heap is a complete binary tree as shown in Figure 1, the root node is the smallest node. Every child node must be bigger than its parent node. The element enters the end of the queue first and compares with its parent node, then adjusts its position until it is larger than the parent node and smaller than its child node. Thus, elements will be placed by priority in the queue.

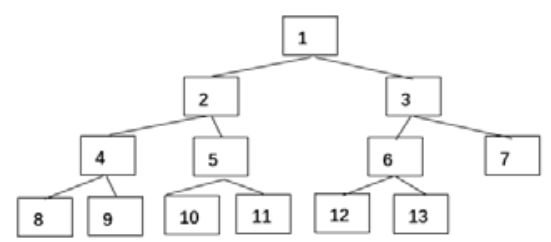

FIGURE I. THE COMPLETE BINARY TREE HAS 4 LAYERS, THE NUMBER OF NODES FROM THE FIRST LAYER TO THE THIRD
LAYER IS THE MINIMUM, AND NODES IN THE FOURTH LAYER ARE ARRANGED FROM LEFT TO RIGHT IN TURN.

The priority queue $p_{-}$queue in (1) is a template class with three template parameters: element type, container type, and comparison operator. pair is the element type which includes the ID number of the nano-node and the packet transmitted by the nano-node. The new comparison operator $\mathrm{cmp}$ is defined to make sure that the packets generated by more than one priority nano-nodes be arrayed based on nano-nodes' ID numbers and queued in the order of its own ID numbers when nano-nodes have the same priority. Apparently, the new comparison operator has two parameters the same as pair. The PQ algorithm is presented in algorithm 1.

$$
\text { priority_queue }\langle\text { pair,vector }\langle\text { pair }\rangle, \text { cmp }\rangle \quad p_{-} q u e u e ;
$$

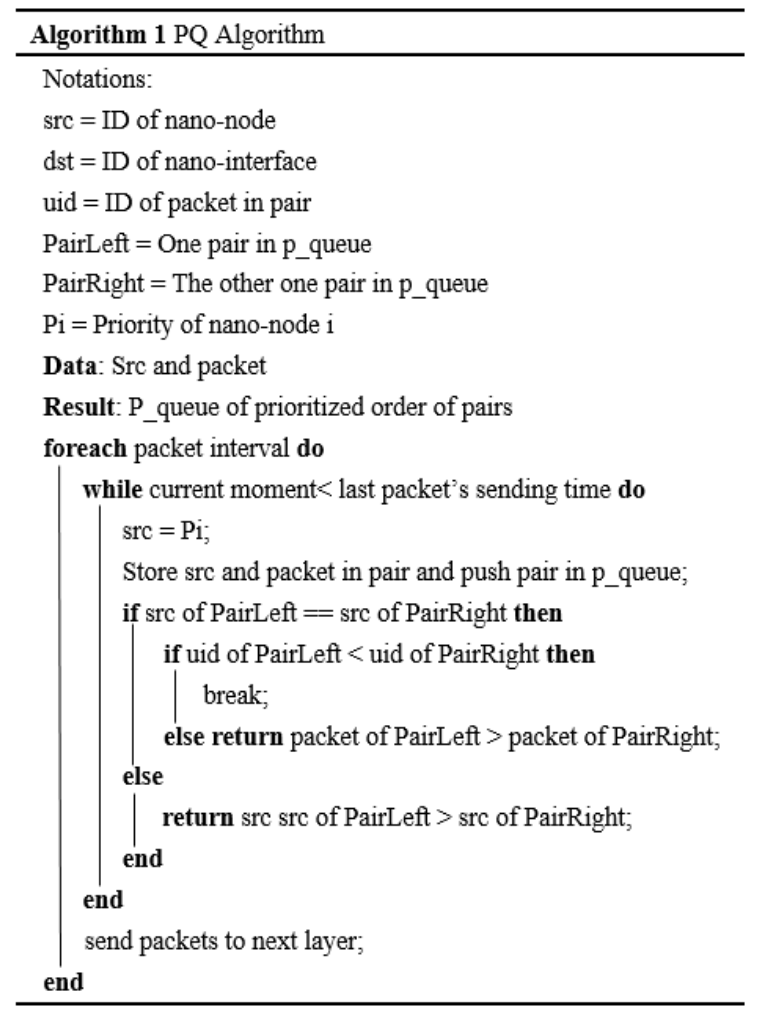

\section{EVALUATION INDEX}

\section{A. Average Transmission Delay}

The average transmission delay of each packet refers to the average time during which a packet is generated by the nanonode and received successfully by the nano-interface, including the queue delay, the transmission delay in the physical layer, the propagation delay in the channel and the reception delay, under the circumstance of ignoring the random delay in packet generation. Meanwhile the transmission delay and the reception delay of each packet are equal. Thus the average transmission delay can be expressed by, 


$$
D_{a i}=D_{q i}+D_{t x}+D_{t r i}+D_{r x}, i=0,1,2, \ldots, N-1
$$

where $D_{q i}$ is the queue delay of the nano-node $i$, standing for the time of processing packets by using the $\mathrm{PQ}$ algorithm during the period of packet interval. $D_{t x}$ and $D_{r x}$ represent the transmission and reception time respectively for each packet, it is calculated to be,

$$
D_{t x}=D_{r x}=\left(N_{b i t}+\text { PhyHead }-1\right) T_{p}+N_{b i t} T_{s}
$$

where $N_{b i t}$ is the number of bits per packet, PhyHead refers to the guard interval of physical layer which ensures that there is enough time to transmit or receive all bits of per packet. $T_{p}$ and $T_{s}$ represent the pulse interval and pulse duration respectively under the TS-OOK modulation scheme. The $D_{t r i}$ in (2) stands for the transmission time in medium from the transmitter to the receiver, which can be computed in (4), $C\left(\mathrm{r}_{i}\right)_{\text {skin }}$ and $C\left(\mathrm{r}_{i}\right)_{\text {skin }}$ in (5), (6) are the information rate in [13] of the nano-node according to Shannon Limit Theorem [14] by taking the TS-OOK communication scheme into account.

$$
D_{t r i}=\frac{N_{b i t}}{C\left(r_{i}\right)}+N_{b i t} \frac{r_{i}}{c}, i=0,1,2, \ldots, N-1
$$

where $r_{i}$ is the distance between the nano-node $i$ and the nano-interface.

$$
\begin{aligned}
& C\left(\mathrm{r}_{i}\right)_{\text {skin }}=2.6 \times 10^{9} r_{i}^{2}-2.3 \times 10^{10} r_{i}+10^{12} \\
& C\left(\mathrm{r}_{i}\right)_{\text {fat }}=2.7 \times 10^{9} r_{i}^{2}-2.2 \times 10^{10} r_{i}+10^{12}
\end{aligned}
$$

\section{B. Network Average Throughput}

Network average throughput means the number of bits successfully received by the nano-interface per second, and can be described by

$$
T P S=\sum_{i=1}^{N_{I D}} M_{i} / D_{a i} / N_{I D}
$$

where $N_{I D}$ means the total number of active nano-nodes transmit packets, $M_{i}$ is the number of bits successfully received in $D_{a i}$ time.

\section{SimUlation RESUlts}

\section{A. Simulation Parameters}

The communication scenario is shown in Figure 2 based on the aforementioned nano-network model with all nano-sensors located in specific position.

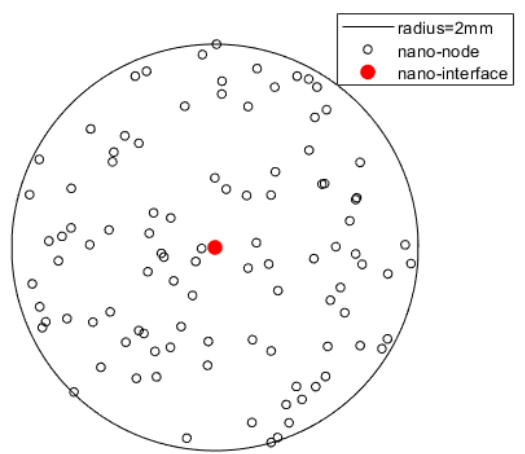

FIGURE II. THE COMMUNICATION SCENARIO.

This paper adopts Network Simulator 3 (NS3) to verify the performance of PB-MAC protocol, first of all, we make all nano-nodes in this nano-network transmit packets with one priority under two protocols in simulation, then divide all nanonodes into three kinds of priorities which range from 1 to 3 then from 3 to 1, last evaluate the performance of PB-MAC protocol by observing the average transmission time and network average throughput in two human tissues and comparing it with transparent transmission MAC protocol. Simulation parameters are shown in Table 2.

TABLE II. SIMULATION PARAMETERS.

\begin{tabular}{|c|c|}
\hline Parameter & Value \\
\hline Number of nano-nodes & 100 \\
\hline Radius of the circle r & $0.002 \mathrm{~m}$ \\
\hline Pulse interval Tp & $10 \mathrm{ps}$ \\
\hline Pulse duration Ts & $100 \mathrm{fs}$ \\
\hline Pulse energy & $1 \mathrm{pJ}$ \\
\hline Packet interval & $0.1 \mathrm{~s}$ \\
\hline Packet size & $102 \mathrm{bytes}$ \\
\hline Communication range of nano-nodes & $0.002 \mathrm{~m}$ \\
\hline Communication range of nano-interface & $0.02 \mathrm{~m}$ \\
\hline Bandwidth B & $1 \mathrm{THz}(0.5 \sim 1 \mathrm{Thz})$ \\
\hline PhyHead & $16 \mathrm{bits}$ \\
\hline Simulation duration & $3 \mathrm{~s}$ \\
\hline
\end{tabular}

\section{B. Average Transmission Delay}

Figure 3 shows the relationship between average transmission delay and 100 nano-nodes' ID. The average transmission delay of nano-node is the average transmission time of packets in thirty packet intervals and it is 0 under the circumstance that all packets of transmitted by the nano-node is failed. It's obvious that average transmission delay in human skin is higher than that in fat no matter what kind of protocol is adopted when all the nano-nodes in this nano-network are active and transmit packets with the same priority. That is because water concentration of human skin is higher than fat and the transmission delay increases with the molecular absorption contributed by the molecules of water vapour. It is come to the conclusion that the average delay of PB-MAC in 
the case of the all active nano-nodes with same priority is almost the same as transparent MAC. Since the packets are first generated first numbered, the PQ algorithm only judge the IDs of packets arriving in chronological order when the nano-nodes have the same priority without sorting. In other words, the proposed PQ algorithm is simple and efficient.

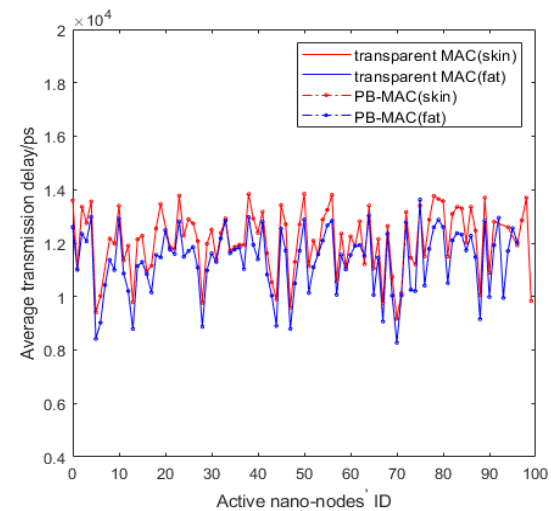

FIGURE III. AVERAGE TRANSMISSION DELAY VS ACTIVE NANONODES' ID FOR TWO HUMAN TISSUES.

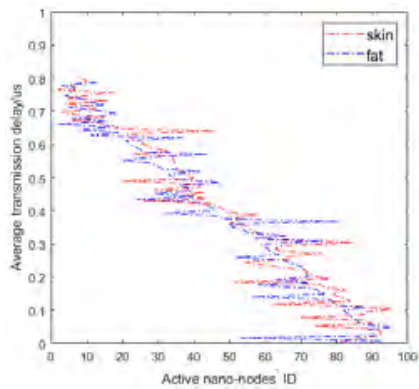

(A)

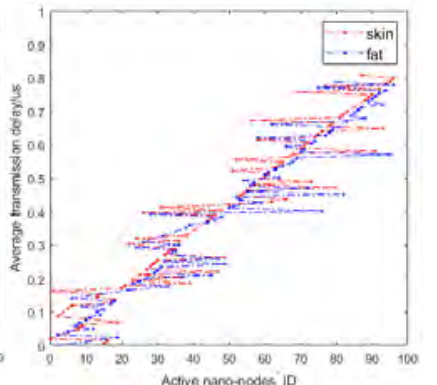

(B)
FIGURE IV. AVERAGE TRANSMISSION DELAY VERSUS DIFFERENT PRIORITIES OF NANO-NODES, WHERE (A) 0 19 (20\%) TRANSMIT P1, 20 49 (30\%) TRANSMIT P2, 50 99 (50\%) TRANSMIT P3 AND (B) 0 19 (20\%) TRANSMIT P3, $20 \sim 49$ (30\%) TRANSMIT P2, 50 99 (50\%) TRANSMIT P1.

It's assumed that nano-nodes transmit data at a random time about a few femtoseconds during simulation period, then we turn the data priority order in turn. The effect of the priorities of nano-nodes on the average transmission delay inside human tissues is illustrated in Figure 4. It is presented that the average transmission delay by adopting PB-MAC protocol increase with the nano-nodes' priorities decrease, this is because that all packets in each packet interval queued by priorities of nanonodes' first then packets' IDs in each priority, and last sent one by one in sequence under the situation that all packets in this priority queue must wait until the last packet is transmitted, which means that packets sent at the MAC layer in order of priority to the next layer. It comes to the conclusion that the higher the priority of data, the smaller the average transmission delay. Meanwhile, the average transmission delay in two human tissues are very close under two priority sequences owing to that the transmission range is the main reason for transmission delay. And it is the same result when we turn the priority order in turn, but the lost packets are different in two situations because of the random channel noise.

\section{Network Average Throughput}

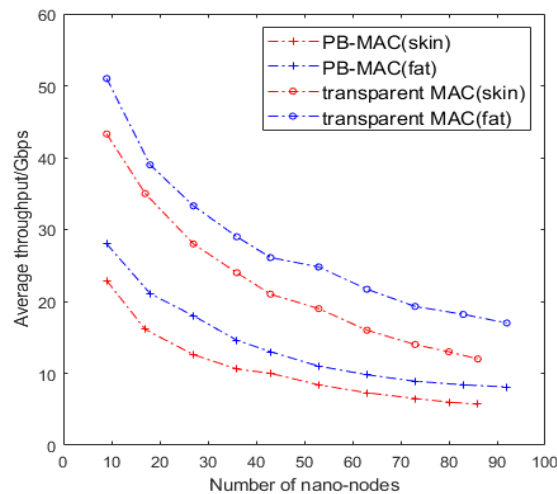

FIGURE V. AVERAGE THROUGHPUT OF PB-MAC PROTOCOL VERSUS NUMBER OF NANO-NODES WHICH TRANSMIT DATA WITH THREE PRIORITIES LIKE FIGURE 4.

In Figure 5, the average throughput is shown as a function of number of nano-nodes, which is smaller in human skin than in fat under two kinds of protocols because of the longer transmission delay, and it decreases as the number of nanonodes increases in two human tissues. Quite evidently, the average throughput of transparent MAC protocol is higher than that of PB-MAC protocol in two human tissues because of the higher average transmission delay.

\section{CONCLUSIONS}

In this paper, we first divide traffic in health monitoring system of iWNSNs into three priorities by taking life-critical data into consideration. Then the system model is demonstrated. Subsequently, we propose the PB-MAC protocol based on the PQ algorithm. The performance of the PB-MAC protocol is comparatively illustrated from the average transmission delay and average throughput, showing that the performing medium of skin is worse than that of fat because of higher water concentration. When all nano-nodes transmit packets with one priority, the obtained results indicate that the average transmission delay of the PB-MAC protocol is almost equal to the transparent MAC in two tissues. Moreover, it's analytically shown that the packets are sent in order of priority to physical layer by changing the priority order of nano-nodes. In a word, the PB-MAC protocol is suitable for health monitoring system with priority requirement for data transmission in in-vivo nanonetworks.

\section{ACKNOWLEDGMENT}

This study is supported in part by the National Natural Science Foundation of China under Grant No.61202384 and the Fundamental Research Funds for the Central Universities under Grant No.22120170186.

\section{REFERENCES}

[1] Hongzhi GUO, Pedram Johari, J. M. Jornet and Zhi SUN, "Intra-Body Optical Channel Modeling for In Vivo Wireless Nanosensor Networks," IEEE Transaction on Nanobioscience, vol. 15, no. 1, pp. 41-52, January 2016. 
[2] Jornet J M, Akyildiz I F. Graphene-based Plasmonic Nano-Antenna for Terahertz Band Communication in Nanonetworks[J]. IEEE Journal on Selected Areas in Communications, vol. 31, no. 12, pp. 685-694, 2013.

[3] Q. Abbasi et al., "Nano-communication for biomedical applications: A review on the state-of-the-art from physical layers to novel networking concepts,’’ IEEE Access J., vol. 4, pp. 3920-3935, 2016.

[4] F. Akyildiz and J. M. Jornet, “The Internet of nano-things," IEEE Wireless Commun., vol. 17, no. 6, pp. 58-63, Dec. 2010.

[5] E. Zarepour, M. Hassan, C. T. Chou, and S. Bayat, "Performance analysis of carrier-less modulation schemes for wireless nanosensor networks,'’ in Proc. IEEE 15th Int. Conf. Nanotechnol. (IEEE-NANO), Nov. 2015, pp. 45-50.

[6] R. ZHANG, K. YANG, A. Alomainy, Q. H. Abbasi, K. Qaraqe, and R. M. Shubair, "Modelling of the terahertz communication channel for invivo nano-networks in the presence of noise," in Proc. 16th Medit. Microw. Symp. (MMS), Jun. 2016, pp. 1-4.

[7] R ZHANG, K YANG, Q. H. Abbasi, K. Qaraqe, and A. Alomainy. Analytical Characterisation of the Terahertz In-vivo Nano-network in the Presence of Interference based on TS-OOK Communication Scheme[J]. IEEE Access, 2017.

[8] Jin SHUAI, Weixia ZOU, Zheng ZHOU, "Priority-based Adaptive Timeslot Allocation Scheme for Wireless Body Area Network,” in Proc. 13th International Symposium on Communications and Information Technologies (ISCIT), 2013, pp. 609-614.

[9] S.Jardosh, P.Ranjan, D.Rawal, ”Prioritized IEEE 802.15.4 for wireless sensor networks," Proc. of the 6th Conference on Wireless Advanced, London, 2010.

[10] K. A. Ali, J. H. Sarker,H. T. Mouftah, ”Urgency-based MAC protocol for wireless sensor body area networks," Proc. of IEEE International Conference on Communications Workshops, Cape Town, South Africa, pp.1-6, 2010.

[11] Y. ZHANG, G. Dolmans, "Priority-guaranteed MAC protocol for emerging wireless body area networks," In: Annals of Telecommunications, vol.66, pp.229-241, April 2011.

[12] Sudip Misra and Subhadeep Sarkar, "Priority-Based Time-Slot Allocation in Wireless Body Area Networks During Medical Emergency Situations: An Evolutionary Game-Theoretic Perspective,” IEEE Journal of biomedical and health informatics, vol. 19, no.2, March 2015.

[13] Zhang R, Yang K, Abbasi Q H, et al. Analytical modelling of the effect of noise on the terahertz in-vivo communication channel for bodycentric nano-networks[J]. Nano Communication Networks, 2017, 15.

[14] C.E. Shannon, A mathematical theory of communication, ACM SIGMOBILE Mob. Comput. Commun. Rev. 5 (1) (2001) 3-55. 